\title{
Evaluation of Disclusion Time in Various Angle's Malocclusions by T-Scan III System
}

\author{
Titirat Chutchalermpan ${ }^{1}$ Jittima Pumklin ${ }^{2}$ Thosapol Piyapattamin ${ }^{3}$
}

\author{
${ }^{1}$ Ban Na Hospital, Amphoe Banna, Nakhon Nayok, Thailand \\ 2Department of Restorative Dentistry, Faculty of Dentistry, \\ Naresuan University, Phitsanulok, Thailand \\ ${ }^{3}$ Department of Preventive Dentistry, Faculty of Dentistry, Naresuan \\ University, Phitsanulok, Thailand
}

\begin{abstract}
Address for correspondence Thosapol Piyapattamin, DDS, PhD, Dip. Thai Board of Orthodontics, Department of Preventive Dentistry, Faculty of Dentistry, Naresuan University, Phitsanulok 65000, Thailand (e-mail: thosapolp@nu.ac.th).
\end{abstract}

Eur J Dent 2019;13:510-513

\begin{abstract}
Keywords

- Angle's malocclusions

- disclusion time

- T-Scan III

Objective The main purpose of this article is to evaluate the disclusion time (DT) in the lateral excursions and protrusion among subjects with Angle's malocclusions by using a T-Scan III system.

Materials and Methods One hundred subjects with malocclusions were divided into Class I, Class II division 1, Class II division 2, and Class III ( $n=25$ per group). All groups' DT of each excursion was evaluated by a T-Scan III system.

Statistical Analysis One-way analysis of variance, followed by a post hoc test, was used to analyze the numerical data at $p<0.05$.

Results In the respective malocclusions, means \pm standard deviations in second(s) of the DT were $2.08 \pm 0.65,2.13 \pm 0.74,2.12 \pm 0.72$, and $3.19 \pm 1.34$ during left excursion; $2.15 \pm 0.94,2.58 \pm 1.16,2.37 \pm 1.07$, and $3.28 \pm 1.25$ during right excursion; and 1.88 $\pm 0.99,2.08 \pm 1.11,2.07 \pm 0.68$, and $3.01 \pm 1.53$ during protrusion. When compared to Class I and Class II malocclusions, Class III showed a significantly higher mean DT of each excursion $(p<0.05)$.

Conclusion Class III had the significantly highest mean DT of each excursion and the significantly longest DT in all excursions.
\end{abstract}

\section{Introduction}

The orthodontic therapeutic goal is an establishment of ideal or normal occlusion, ${ }^{1}$ comprising static occlusion ${ }^{2,3}$ and function aspects of the occlusion. ${ }^{4}$ Dental occlusion is an important factor that determines the masticatory performance. ${ }^{1}$ Subjects with normal occlusion have a better masticatory performance than those with malocclusions. ${ }^{5}$ Class II and III relationships are reported to be predisposing factors to the contribution of temporomandibular disorder (TMD) ${ }^{6,7}$ Maxillary anterior teeth's inclination affects condylar position and movement during protrusion and their lingual inclinations in Class II Division 2 induce a posterior position of the condyle. ${ }^{8,9}$ However, an association between malocclusions and TMD has long been debated. ${ }^{10,11}$

In addition to the dental alignment, disclusion time (DT), the duration of time starting after mandibular movement from maximum intercuspation to excursive movement until all posterior teeth's disclusion and anterior teeth's contacts are observed, ${ }^{12}$ is considered a TMD-related factor. Lack of anterior guidance in Class II and III subjects with anterior crossbite causes a long contact among their posterior teeth during protrusion, ${ }^{9,13}$ and a prolonged duration of occlusal contact affects the amount of load distribution on the masticatory system. ${ }^{14}$ However, DT among malocclusion types has not been clearly reported.

A T-Scan I system has been firstly used to measure DT..$^{15}$ With an improved speed of recording and reporting the data in a 0.003 seconds time increment, the T-Scan III system (Tekscan, Boston, Massachusetts, United States) is the device's newest version for computerized occlusion analyses. ${ }^{16}$ The T-Scan III system has been widely used in dentistry, due to its advantages in measuring the force-time sequences in mandibular static and dynamic positions. ${ }^{17}$

TMD is reported to relate to some occlusal features, that is, premature occlusal contacts, Class II and III relationships, 
and over 5.0-mm overjet or overbite. ${ }^{6,7,18}$ Among those with malocclusions, TMD subjects are reported to possess a longer DT than non-TMD ones. ${ }^{13}$ Despite the controversy of the possible effects of Angle's Class II and III on TMD, their DT is hypothesized to differ from that of Class I. Hence, the aim of this study was to compare the DT during mandibular lateral excursions and protrusion among the subjects with different Angle's malocclusions by using a T-Scan III system.

\section{Materials and Methods}

This study was approved by Naresuan University Ethical Committee (IRB Number 0721/60). Post-explanation of the procedures, written inform consents were obtained from all subjects. An initial screening and a clinical examination were performed to exclude the subjects with either of these criteria, that is, restoration(s) with dental implant or fixed prosthesis, past or ongoing orthodontic treatment, molar relationship's classification on one side different from the other, TMD, and parafunctional habit. One hundred volunteers were included and presented at least 28 permanent teeth with symmetrical dental arch forms and equal number of teeth. Based on Angle's classification of malocclusions, they were divided into four groups (Class I, Class II division 1, Class II division 2, and Class III; $n=25$ per group).

Each subject participated in two visits for the collections of data. The first one included impression taking for diagnostic maxillary and mandibular models. Mesiodistal width of the maxillary teeth was measured by a digital Vernier caliper (Mitutoyo, Kanagawa, Japan) and recorded into a T-Scan III system, Version 9.1.9 (Tekscan) for transferring individual dental arch dimension. The second one was 7 days later and included the recording procedures of all participants' DT by the same T-Scan III system. With an upright sitting position on a dental chair and their Frankfort horizontal plane parallel to the floor, the subjects were asked to perform mandibular protrusion and movement to lateral sides. The correct motions were repeatedly conducted three times. The T-Scan recording into the T-Scan sensor was done in each excursion, when the subjects were asked to be in maximum intercuspation for 3 to 5 seconds before excursion movement. By selecting left excursion, right excursion, or protrusion in the program, the time difference between points $C$ and $D$ was recorded and designated as DT shown in the graph of force versus time and timing table ( - Fig. 1). DT was measured three times in each excursion and their means and standard deviations (SD) were calculated.

Statistical analyses of the obtained data were performed using PASW Statistics for Windows, Version 17.0 (SPSS, Chicago, Illinois, United States). Standard descriptive statistics were used for calculating means, SD, and range of DT in each excursion (left excursion, right excursion, and protrusion). A one-way analysis of variance, followed by a post hoc analysis by least significant difference (LSD) test, was used for comparing mean DT in each excursion. The level of statistical significance was the value of $p<0.05$.

\section{Results}

Details of the subjects were shown in - Table 1 . There was no significant difference in the subjects' mean age ( $p=0.716)$.

There were intergroup significant differences in the subjects' mean overbite $(p=0.000)$. The significantly highest overbite value was seen in Class II division 2, while the significantly lowest one in Class III. There were intergroup significant differences in the subjects' mean overjet $(p=0.000)$, except for that between Class II division 2 and Class III $(p=0.614)$. The significantly highest overjet value was seen in Class II division 1, while the significantly lowest one in Class III ( - Table 1).

Each excursion's mean DT among the malocclusions was shown in - Table 2. It was observed in all excursions that Class III possessed the significantly longest mean DT, while Class I the shortest one. With respect to the excursions, there were intergroup significant differences in the mean DT (left excursion, $p=0.000$; right excursion, $p=0.003$; and protrusion, $p=0.002$ ). A post hoc LSD test revealed the significantly longest mean DT $(p<0.05)$ in Class III, when compared to those in other malocclusions ( $\boldsymbol{- \text { Fig. }} \mathbf{2}$ ).

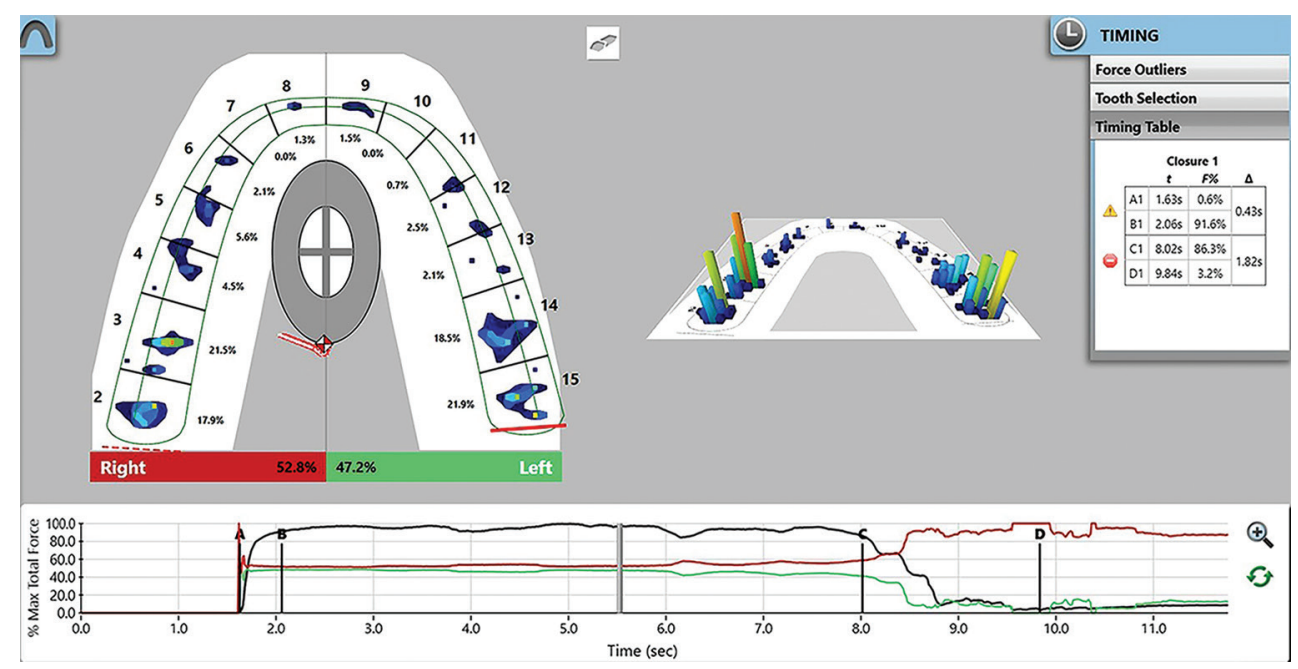

Fig. 1 Timing table with the recorded disclusion time $(\Delta C-D)$ shown in a T-Scan display screen in two (-left) and three (-right) dimensional images using a T-Scan III system. The Universal Numbering System is shown on each maxillary tooth's buccal side. 
Table 1 Sample characteristics in each malocclusion type

\begin{tabular}{|l|l|l|l|l|l|l|l|l|}
\hline \multirow{2}{*}{ Malocclusion $(\boldsymbol{n})$} & \multicolumn{2}{|c|}{ Gender } & \multicolumn{2}{c|}{ Age $(\mathrm{y})$} & \multicolumn{2}{c|}{ Overbite (mm) } & \multicolumn{2}{c|}{ Overjet (mm) } \\
\cline { 2 - 10 } & $\mathrm{F}(\boldsymbol{n})$ & $\mathrm{M}(\boldsymbol{n})$ & Mean \pm SD & Range & Mean \pm SD & Range & Mean \pm SD & Range \\
\hline Class I (25) & 14 & 11 & $21.5 \pm 0.78^{a}$ & $18.0-25.0$ & $2.42 \pm 1.05^{a}$ & $0.50-5.00$ & $2.52 \pm 1.04^{a}$ & $0.50-4.00$ \\
\hline Class II division 1 (25) & 16 & 9 & $22.0 \pm 0.55^{a}$ & $18.0-26.0$ & $3.10 \pm 1.11^{b}$ & $1.00-5.50$ & $4.24 \pm 1.50^{b}$ & $2.00-9.00$ \\
\hline Class II division 2 (25) & 13 & 12 & $23.5 \pm 1.04^{a}$ & $18.0-31.0$ & $4.70 \pm 1.42^{c}$ & $2.50-8.00$ & $1.62 \pm 0.67^{c}$ & $0.50-3.50$ \\
\hline Class III (25) & 16 & 9 & $22.5 \pm 0.44^{a}$ & $18.0-27.0$ & $1.44 \pm 0.95^{d}$ & $0.00-3.50$ & $1.46 \pm 1.11^{c}$ & $(-1.00)-4.00$ \\
\hline$p$-Value & & & $>0.05$ & & $<0.05$ & & $<0.05$ & \\
\hline
\end{tabular}

Abbreviations: F, female; M, male; SD, standard deviation.

Note: Different lower case letters $(a-d)$ indicate intracolumn statistically significant differences $(p<0.05)$.

Table 2 Disclusion time(s) in each excursion among malocclusion types

\begin{tabular}{|l|l|l|l|l|l|l|}
\hline \multirow{2}{*}{ Malocclusion $(n)$} & \multicolumn{2}{|c|}{ Left excursion } & \multicolumn{2}{c|}{ Right excursion } & \multicolumn{2}{c|}{ Protrusion } \\
\cline { 2 - 7 } & Mean \pm SD & Range & Mean \pm SD & Range & Mean \pm SD & Range \\
\hline Class I (25) & $2.08 \pm 0.65$ & $0.50-3.21$ & $2.15 \pm 0.94$ & $0.35-3.68$ & $1.88 \pm 0.99$ & $0.35-4.04$ \\
\hline Class II division 1 (25) & $2.13 \pm 0.74$ & $0.67-3.65$ & $2.58 \pm 1.16$ & $0.90-5.71$ & $2.08 \pm 1.11$ & $0.47-5.00$ \\
\hline Class II division 2 (25) & $2.12 \pm 0.72$ & $1.02-3.52$ & $2.37 \pm 1.07$ & $0.56-4.11$ & $2.07 \pm 0.68$ & $1.17-3.48$ \\
\hline Class III (25) & $3.19 \pm 1.34$ & $0.62-5.63$ & $3.28 \pm 1.25$ & $0.67-5.25$ & $3.01 \pm 1.53$ & $1.01-6.29$ \\
\hline
\end{tabular}

Abbreviation: SD, standard deviation.

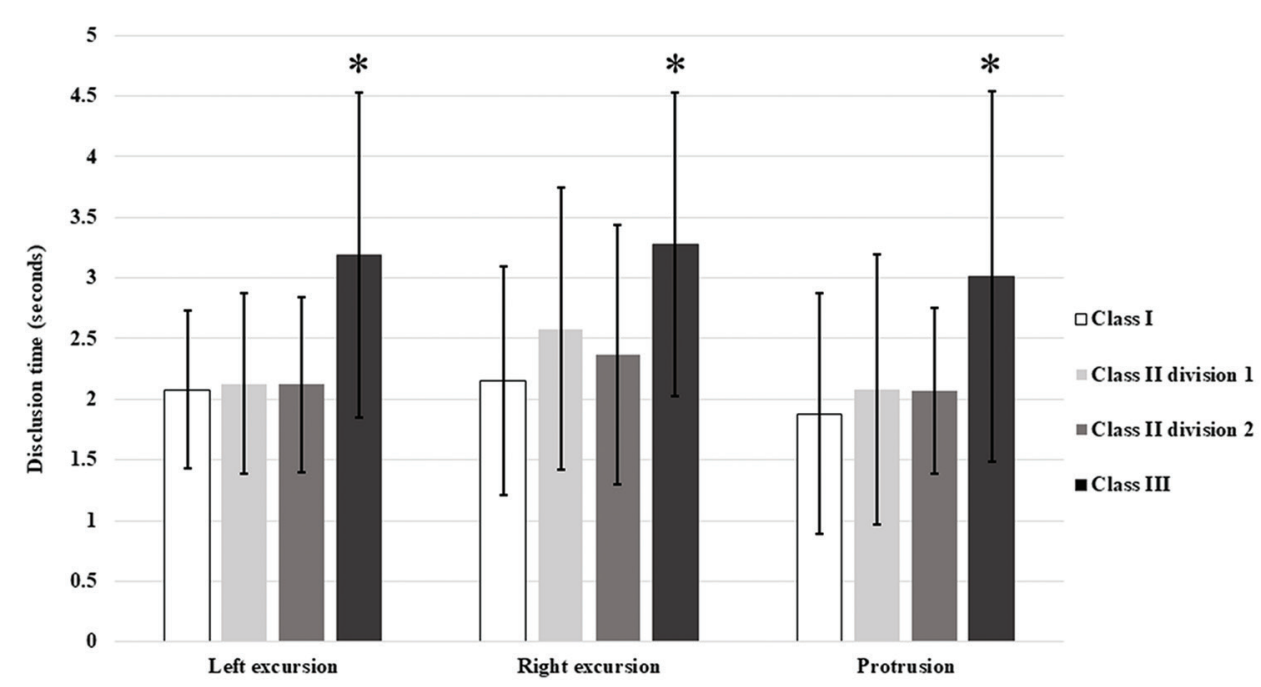

Fig. 2 Mean disclusion time of each excursion among malocclusion types. Asterisks $\left(^{*}\right)$ indicate statistically significant differences $(p<0.05)$.

\section{Discussion}

DT is utilized for some investigations into the muscular activities and masticatory performance, and the DT over 0.5 seconds can elevate the contraction levels of temporalis and masseter muscles. ${ }^{13,16} \mathrm{~A}$ prolonged DT leads to masticatory muscles' higher electromyography activity and abnormal stress distributions in the articular disc, thus facilitating TMD symptoms' occurrences. ${ }^{6,19}$ A successful method for reducing the symptoms is shortening the $\mathrm{DT}^{13}$ and a reduction in DT to less than 0.5 seconds per excursion has been reported to decrease muscular hyperactivity and their related symptoms. ${ }^{19,20}$ In spite of the fact that the prolonged DT (over $0.5 \mathrm{~s}$ ) of all excursions was detected in our non-TMD subjects, it was explicable by a phenomenon of physiologic tolerance that an individual might have an adaptive capability to malocclusion and functional appearances. ${ }^{21}$

During excursions, there should be incisal contacts, which are determined by overbite and overjet, to facilitate an achievement of the anterior guidance and to cause an immediate disclusion of the posterior teeth. ${ }^{22}$ Class II malocclusion was reported to have the longest DT, followed 
by Class III and Class I malocclusions, respectively. ${ }^{13}$ In this study, Class III malocclusion showed the significantly longest DT of all excursions, followed by Class II division 1, Class II division 2, and Class I malocclusions, respectively. Discrepancies between the above results might have contributed to our larger sample size and usage of the T-Scan system's newer version, apart from the subjects' racial group and age.

Some relationships between overjet and DT have been shown that subjects with an overjet larger than $3 \mathrm{~mm}$ result in a prolonged DT. ${ }^{23}$ However, no intergroup significant difference in the DT between Class I and Class II malocclusions (except for those between Class III malocclusions) was detected in our study, despite all intergroup significant differences in the overjet (except for that between Class II division 2 and Class III malocclusions) and the overbite. Our findings illustrated that neither overjet nor overbite affected the DT. Some further investigation is needed to clarify such inconsistency.

Dental and skeletal characteristics are related to signs and symptoms of TMD. Not only the dental factors but also the craniofacial skeletal structures are reported to be among TMD contributing factors. ${ }^{8,9,24}$ Associations between various morphological occlusions and TMD, including a prolonged DT, have long been debated. Although some significant DT differences in Class III malocclusion, lack of overjet- and overbite-DT relationships, and some possibilities of detecting the prolonged DT in non-TMD subjects were shown in our study, it focused solely on Angle's classifications and was unable to relate such findings with the skeletal structures. Consequently, it is necessary to prove a correlation, if any, between DT and TMD subjects with various skeletal patterns.

\section{Conclusion}

When compared to Class I and II malocclusions, Class III possessed the significantly highest mean DT of each excursion and the significantly longest DT in all excursions. Different excursions caused no intragroup significant difference in their mean DT.

\section{Funding}

This study was supported by The Research Funds for Graduate Students from Faculty of Dentistry, Naresuan University.

\section{Conflict of Interest}

None declared.

\section{References}

1 Al-Nimri KS, Bataineh AB, Abo-Farha S. Functional occlusal patterns and their relationship to static occlusion. Angle Orthod 2010;80(1):65-71

2 Angle EH. Classification of malocclusion. Dent Cosmos 1899;41:248-264

3 Andrews LF. The six keys to normal occlusion. Am J Orthod 1972;62(3):296-309

4 Roth RH. The maintenance system and occlusal dynamics. Dent Clin North Am 1976;20(4):761-788
5 Proffit WR, Fields HW. Contemporary Orthodontics. 5th edition. St. Louis: Mosby; 2013:124-126

6 Selaimen CM, Jeronymo JC, Brilhante DP, Lima EM, Grossi PK, Grossi ML. Occlusal risk factors for temporomandibular disorders. Angle Orthod 2007;77(3):471-477

7 Chisnoiu AM, Picos AM, Popa S, et al. Factors involved in the etiology of temporomandibular disorders - a literature review. Clujul Med 2015;88(4):473-478

8 Stringert HG, Worms FW. Variations in skeletal and dental patterns in patients with structural and functional alterations of the temporomandibular joint: a preliminary report. Am J Orthod 1986;89(4):285-297

9 Hwang CJ, Sung SJ, Kim SJ. Lateral cephalometric characteristics of malocclusion patients with temporomandibular joint disorder symptoms. Am J Orthod Dentofacial Orthop 2006;129(4):497-503

10 Luther F. TMD and occlusion part II. Damned if we don't? Functional occlusal problems: TMD epidemiology in a wider context. Br Dent J 2007;202(1, E3):E3, discussion 38-39

11 Runge ME, Sadowsky C, Sakols EI, BeGole EA. The relationship between temporomandibular joint sounds and malocclusion. Am J Orthod Dentofacial Orthop 1989;96(1):36-42

12 Ramfjord SP. The role of occlusion in temporomandibular joint and associated muscle disorders. J Gnathol 1990;9:23-31

13 Kerstein RB. Disclusion time measurement studies: a comparison of disclusion time between chronic myofascial pain dysfunction patients and nonpatients: a population analysis. J Prosthet Dent 1994;72(5):473-480

14 Kerstein RB. A comparison of traditional occlusal equilibration and immediate complete anterior guidance development. Cranio 1993;11(2):126-139, discussion 140

15 Maness WL, Benjamin M, Podoloff R, Bobick A, Golden RF. Computerized occlusal analysis: a new technology. Quintessence Int 1987;18(4):287-292

16 Kerstein RB, Lowe M, Harty M, Radke J. A force reproduction analysis of two recording sensors of a computerized occlusal analysis system. Cranio 2006;24(1):15-24

17 Trpevska V, Kovacevska G, Benedetti A, Kanurkova L. Occlusion timeline analyses with T-Scan III system in subjects with neutroocclusion. Int J Sci Res (Ahmedabad) 2017;6(3):66-69

18 Pullinger AG, Seligman DA, Gornbein JA. A multiple logistic regression analysis of the risk and relative odds of temporomandibular disorders as a function of common occlusal features. J Dent Res 1993;72(6):968-979

19 Thumati P, Manwani R, Mahantshetty M. The effect of reduced disclusion time in the treatment of myofascial pain dysfunction syndrome using immediate complete anterior guidance development protocol monitored by digital analysis of occlusion. Cranio 2014;32(4):289-299

20 Thumati P, Thumati RP. The effect of disocclusion timereduction therapy to treat chronic myofascial pain: a single group interventional study with 3 year follow-up of 100 cases. J Indian Prosthodont Soc 2016;16(3):234-241

21 Okeson JP, Management of Temporomandibular Disorders and Occlusion. 7th edition. St. Louis: Mosby; 2013:443-459

22 Al-Hiyasat AS, Abu-Alhaija ES. The relationship between static and dynamic occlusion in 14-17-year-old school children. J Oral Rehabil 2004;31(7):628-633

23 Oltramari PV, Conti AC, Navarro RdeL, Almeida MR, Almeida-Pedrin RR, Ferreira FP. Importance of occlusion aspects in the completion of orthodontic treatment. Braz Dent J 2007;18(1):78-82

24 Almăşan OC, Băciuț M, Almăşan HA, et al.Skeletal pattern in subjects with temporomandibular joint disorders. Arch Med Sci 2013;9(1):118-126 